\title{
Changes of heat pain sensitivity during the menstrual cycle in Japanese young adults: a randomized trial
}

\author{
Takahiro Kakeda ${ }^{1}$, Kei Takani ${ }^{2}$, Koichi Takaoka ${ }^{1}$, \\ Noriyoshi Tanaka ${ }^{3}$, and Yuichi Ogino ${ }^{4}$ \\ ${ }^{1}$ Department of Nursing, Faculty of Nursing, Kansai University of Social Welfare \\ ${ }^{2}$ Division of Nursing, Matsue Red Cross Hospital \\ ${ }^{3}$ Department of Nursing, Faculty of Nursing, University of Shizuoka \\ ${ }^{4}$ Department of Anesthesiology, Graduate School of Medicine, Gunma University
}

\begin{abstract}
The aim of this study was to compare pain sensitivity between the follicular and luteal phases of the menstrual cycle in Japanese young adults. Fifteen females participated in this randomized control trial with crossover design. The pain threshold (defined as the temperature when the subject started to feel pain) was measured using a heat pain stimulator on the short flexor muscle of the thumb. For each subject, the stimulus was performed 10 times, alternating between the right and left hand. Pain reactivity was evaluated using a self-identified threshold of heat pain, salivary alpha amylase, emotional evaluation using a profile of mood states and visual analog scale scores. After comparative analysis, we found that pain threshold under the follicular phase was significantly increased compared to that under the luteal phase, indicating that the menstrual cycle would influence the severity of pain perception. Pain sensitivity under the luteal phase is more sensitive to nociceptive stimuli than those under the follicular phase. We therefore need to conduct pain management with paying heed to the menstrual cycle. Additionally, young women in reproductive-age should be more protective in pain management during the luteal phase of their menstrual cycle.
\end{abstract}

Keywords

Female adults; Heat pain; Menses; Pain stimulus

Received: 26 July 2019

Accepted: 5 November 2019 


\section{Introduction}

Gonadal hormones including estrogen and progesterone regulate the menstrual cycle and have an important role in the experience of pain in healthy women ${ }^{12}$. During the menstrual cycle, responses to experimental pain stimuli and pain sensitivity in the luteal phase are higher compared to those in the follicular phase ${ }^{11)}$. Although the underlying mechanisms for these effects on nociception remain unclear, it is possible that fluctuation of gonadal hormones such as estradiol and progesterone influence edogenous pain modulation system ${ }^{2}$. In contrast, few studies have examined the differences in pain sensitivity during the menstrual cycle, specifically in Japanese young adults. Therefore, the aim of this study was to examine whether pain sensitivity fluctuates between the follicular and luteal phases during the menstrual cycle in Japanese young adult females.

\section{Materials and Methods}

\section{Ethical consideration}

This study was approved by the ethics committee of the Kawasaki University of Medical Welfare in Japan. Also, subjects signed a consent form after receiving an explanation of the purpose and procedures of this study.

\section{Study design}

This study was designed as a single-blind and randomized crossover-controlled trial. For the purpose of this study, the follicular phase of the menstrual cycle was defined as the period within 1 week after last day of menses and the luteal phase was defined as the period within 1 week before a scheduled menstrual day.

\section{Subjects}

Fifteen Japanese young adults with a regular menstrual cycle participated in this study (ages $21.3 \pm 0.18$ yr). Subjects were recruited to participate through billboard advertisements at a university campus. The inclusion criteria were being pain-free, having a regular menstrual cycle, and being nonsmokers. None of the subjects had a history of neurological disorders, cardiovascular disease, external injuries, respiratory or autonomic dysfunction, or had been on medication recently. Subjects were asked to refrain from ingesting any food or drink for at least $3 \mathrm{~h}$ and to abstain from alcohol for at least $24 \mathrm{~h}$ before the study. In addition, subjects were to avoid intense exercise the day before the research. In this study, none of the subjects demonstrated anything unusual or had any adverse event.

\section{Determination of the menstrual cycle phase}

Each subject was tested twice on two occasions during their menstrual cycle, once in the follicular phase (defined as days 1-7 after the stop of menses) and once in the luteal phase (defined as days 1-7 before the estimated start-day of menses).

\section{Experimental heat pain stimulus}

We employed the heat pain stimulator (UDH105, Unique medical company, Tokyo, Japan), which was a probe $18 \mathrm{~mm}$ in diameter, to generate experimental pain on the short flexor muscle of thumb. During the heat pain stimulus, we measured the subject's pain threshold (defined as the temperature when the subject started to feel heat pain induced by the heat pain stimulator). The data was recorded when the subjects pushed a detect-button to indicate pain. For the subject's safety, the heat pain stimulation 
stopped after the pain threshold was reached. In addition, the heat pain stimulator probe was limited $60^{\circ} \mathrm{C}$, regardless of whether the subject reported reaching the pain threshold. Surface temperature on the probe was increased at $0.25^{\circ} \mathrm{C}$ intervals per second starting from $30^{\circ} \mathrm{C}$. The heat pain stimulus was performed 10 times in total, alternating between the right and left hands of the subjects with each repetition. Furthermore, subjects rested for 2 min between each heat pain stimulus to avoid any carryover effects and habituation to the heat pain stimulus.

\section{Research indexes}

\section{Pain threshold}

During the heat pain stimulus, pain threshold was defined as the temperature when the subject first started to feel heat pain induced by the heat pain stimulator. Subjects indicated the initial onset of pain by pushing a button to indicate pain threshold.

\section{Subjective estimation of heat pain using the visual analogue scale}

The visual analogue scale (VAS) of heat pain intensity was used to self-evaluate pain intensity (range, $0-10$ ). The VAS of pain intensity was a $10 \mathrm{~cm}$ linear scale with no numerical markings between "not at all painful" (0) at one end and "extremely painful" (10) at the other. Subjects drew a horizontal line on the VAS to evaluate pain intensity immediately upon withdrawing their hand from the pain stimulus.

\section{Salivary alpha-amylase}

The alpha-amylase (AA) in saliva was measured as an indicator of acute stress because of its association with the sympathoadrenal medullary system and its known response to acute stress in humans ${ }^{14)}$. The AA levels for each subject were analyzed by using a portable monitor (Nipro CM-3.1, Osaka, Japan). The meter tip was immersed in saliva under the tongue of the subjects for 30 seconds. The test strip was then read by an optical analyzer and the value was shown on the monitor display. The pain-associated stress was estimated by using the level of AA between pre- and poststress conditions.

\section{Psychological evaluation by Profile of Mood States-Brief Form (POMS)}

Psychological factors such as mood and emotion have known to influence the perception and modulation of pain. This study used the brief Japanese version of the profile of mood state (POMS) for psychological evaluation. The brief POMS is a list of 30 questions which classifies 6 mood subscales (tensionanxiety [T-A], depression-dejection [D], angerhostility $[\mathrm{A}-\mathrm{H}]$, vigor $[\mathrm{V}]$, fatigue $[\mathrm{F}]$, and confusion $[C]$ ). Each subject rated their mood and emotion using a 5 -point scale ranging from "not at all" (0 points) to "quite frequently" (4 points) how they felt "right now" between pre-heat pain stimulus and post-heat pain stimulus. Scores were recorded for pre- and post-stress condition for each phase. Total mood disturbance (TMD) was then calculated by adding the T-A, D, A-H, F and C scores and subtracting the $\mathrm{V}$ score.

\section{Study procedures}

This study was performed in an air-conditioned experimental room (room temperature $25.0 \pm 2.0^{\circ} \mathrm{C}$, humidity $50.3 \pm 2.1 \%$ ). Each subject underwent 2 test sessions during their follicular and luteal phases. The workflow of one test is shown in Figure 1. First, subjects sat on a chair for at least 10 minutes to rest after walking into the experimental room. Subjects were then taken about a past medical history, 


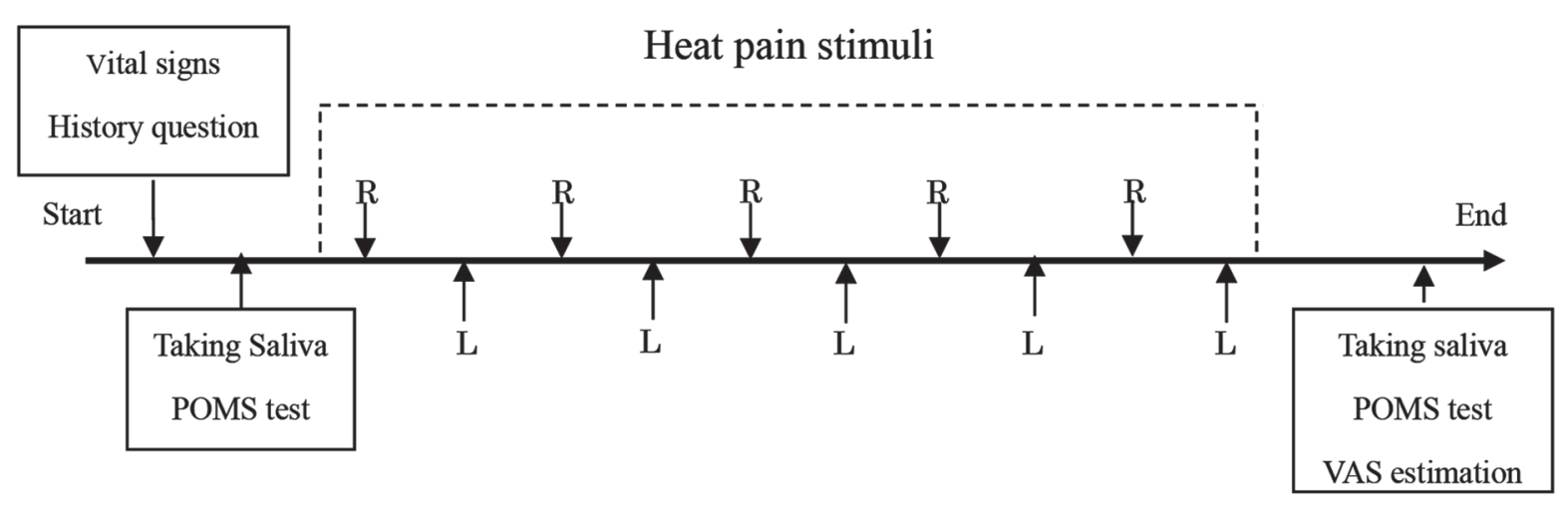

Fig.1 Study protocol.

This study was conducted during the follicular and luteal phases of the menstrual cycle. Heat pain stimuli were performed a total 10 times on the right and left hands, alternately. Moreover, subjects rested for 2 min between each heat pain stimulus to avoid any carryover effects and habituation to the heat pain stimulus. POMS: Profile of Mood States; VAS: Visual analogue scale; R: right hand; L: left hand.

and their vital signs were examined for abnormalities. After the medical interview, the AA value was collected before the experimental condition. Their pre-experimental psychological status was also evaluated by using the POMS. Next, researchers applied heat stimulation to an area marked directly on each flexor pollicis brevis. Subjects pushed the button to declare pain threshold immediately when they sensed heat pain. The probe temperature was recorded as pain threshold. Immediately after the pain stimuli, they evaluated pain intensity using the VAS. Between stimuli, to avoid any carry-over effects and habituation of pain stimuli, the heat pain stimuli were conducted in the right and left hand alternatingly. In addition, the subjects rested for two minutes between each stimulus. After 10 periods of heat stimuli, post-test saliva measurement was taken for AA value and subjects self-evaluated their psychological status using the POMS.

\section{Statistical analysis}

Data were analyzed using SPSS Version 23.0. All data are expressed as mean \pm standard deviation of the mean. Participant characteris- tics were analyzed with descriptive statistics. Value of pain threshold, pain VAS, AA, and psychological status were analyzed using nonparametric sign tests. The criterion for statistical significance was set at 0.05 .

\section{Results}

\section{Pain threshold and pain intensity}

Figure 2 shows the pain threshold temperature during the follicular phase, which was significantly higher compared to that during the luteal phase. During the follicular phase, the temperature associated with the pain threshold was $46.5 \pm 0.1^{\circ} \mathrm{C}$ and during the luteal phase it was $46.0 \pm 0.2^{\circ} \mathrm{C}(p=0.002)$ (Figure 2). The VAS scores did not significantly change between the follicular and luteal phases $(5.2 \pm 2.0$ vs. $4.7 \pm$ 2.0 , respectively; $p=0.180$ ).

\section{Acute stress estimation using the alpha amylase in saliva}

The AA in saliva during the follicular phase significantly decreased between the pre- and post-conditions $(45.1 \pm 45.4 \mathrm{KIU} / \mathrm{L}$ vs.22.1 \pm 


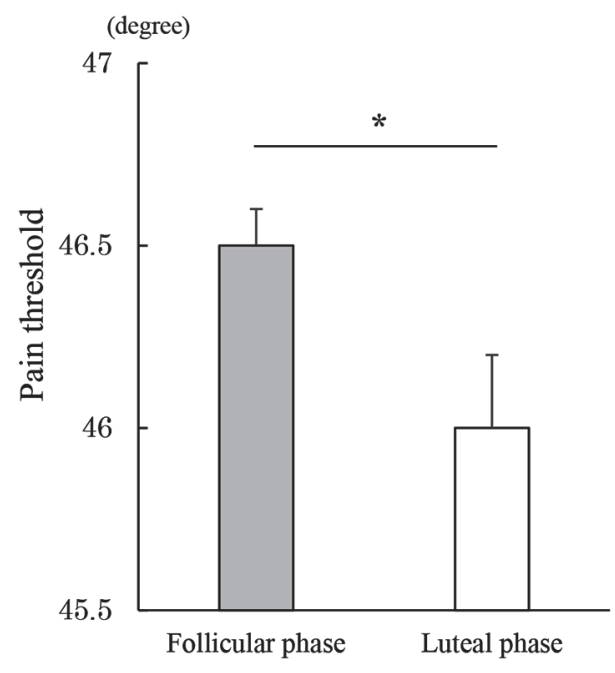

Fig.2 Pain threshold between the follicular and luteal phases by the heat pain stimuli. ${ }^{*} p<0.05$

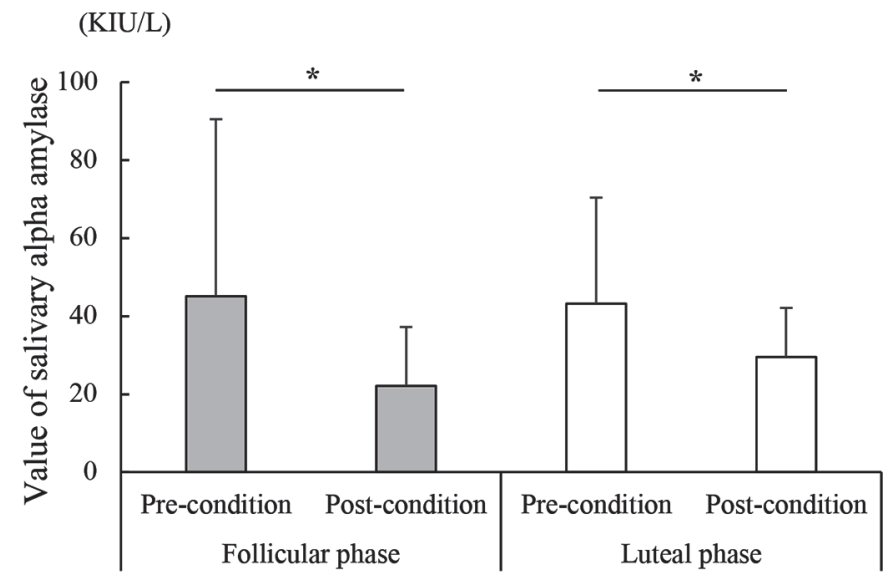

Fig.3 Pre- and post-stimuli changes in alpha amylase values taken from saliva.

${ }^{*} p<0.05$

Table 1 Scores of the Profile of Mood States for each condition

\begin{tabular}{lccccccc}
\hline & \multicolumn{2}{c}{ Follicular phase } & & & \multicolumn{2}{c}{ Luteal phase } & \\
\cline { 2 - 3 } & Pre-condition & Post-condition & & & Pre-condition & Post-condition & \\
\hline Tension-Anxiety & $47.7 \pm 8.6$ & $45.3 \pm 7.7$ & 0.125 & & $46.6 \pm 6.6$ & $45.2 \pm 6.9$ & 0.388 \\
Depression-Dejection & $47.8 \pm 5.7$ & $47.1 \pm 5.9$ & 0.289 & & $48.0 \pm 5.8$ & $48.3 \pm 6.0$ & 0.581 \\
Anger-Hostility & $46.1 \pm 9.8$ & $46.9 \pm 11.2$ & 1.000 & & $47.1 \pm 7.2$ & $45.3 \pm 5.8$ & 0.227 \\
Vigor & $44.8 \pm 8.6$ & $44.3 \pm 8.8$ & 1.000 & & $48.1 \pm 7.1$ & $45.3 \pm 7.6$ & 0.388 \\
Fatigue & $47.5 \pm 8.2$ & $44.9 \pm 9.6$ & 0.180 & & $48.1 \pm 7.8$ & $45.7 \pm 7.2$ & 0.180 \\
Confusion & $51.5 \pm 9.5$ & $49.9 \pm 8.0$ & 0.774 & & $51.5 \pm 10.1$ & $50.4 \pm 8.4$ & 1.000 \\
TMD & $19.1 \pm 11.7$ & $15.9 \pm 11.4$ & 0.065 & & $17.5 \pm 14.0$ & $17.4 \pm 13.5$ & 1.000 \\
\hline
\end{tabular}

TMD: Total mood disturbance

15.1 KIU/L, respectively; $p=0.007)$. The AA in saliva during the luteal phase also significantly decreased between pre-and post-conditions $(43.2 \pm 27.2 \mathrm{KIU} / \mathrm{L}$ vs, $29.5 \pm 12.6 \mathrm{KIU} / \mathrm{L}$, respectively; $p=0.022$ ) (Figure 3 ).

\section{Emotional estimation using the POMS}

The POMS scores for 6 subscales during both the follicular and luteal phases are shown in Table 1. None of the POMS showed significant differences in subscale or TMD scores during the follicular phase. Similarly, the POMS scores during the luteal phase were not significantly different in any of the subscale or TMD scores.

\section{Discussion}

The results of the current study show that pain threshold in response to heat pain during 
the follicular phase is significantly higher than that during the luteal phase in Japanese young adults. These findings indicate that pain perception under the luteal phase is more sensitive compared to that under the follicular phase during the menstrual cycle. These results are consistent with prior studies that show higher pain thresholds for pain perception in the follicular phase ${ }^{1,11,15)}$. In part, these findings also correspond with previous findings that responses to clinical pricking pain due to rocuronium injection ${ }^{3)}$ and propofol injection ${ }^{4)}$ are greater in the luteal phase than in the follicular phase. Moreover, Piroli et al. have shown that healthy young women exhibit lower postoperative pain sensitivity by undergoing gynecological laparoscopy in the follicular phase compared with that in the luteal phase ${ }^{9)}$. Furthermore, results of the present study agree with recent findings that pinprick evoked pain, incision-induced pain, and mechanical hyperalgesia were significantly greater during the luteal phase compared to the follicular phase in female volunteers ${ }^{10}$. Moreover, the VAS scores of pain intensity, in relation to the pain threshold, were not significantly different between the two phases in the present study. As mentioned above, the pain threshold was defined as the time when the subjects started to feel painful heat sensation induced by the heat-pain stimulator. That is, we consider that the subjects did not intentionally tolerate the heat pain stimulus in each phase, respectively. These findings thus indicate the need to provide more preventative pain management during the luteal phase in Japanese women of reproductive-age.

The reasons for differences in pain response between the menstrual phases remain unclear. However, a decline in progesterone production at the late luteal phase induces an increase of prostaglandin E2 and prostaglandin $\mathrm{F}_{2 \alpha}{ }^{6}$.
These prostaglandins induce contractions of the uterine muscle and reinforce egestion of desquamated endometrial membrane, which might lower the pain threshold. Additionally, Lusk et al. ${ }^{8)}$ has reported the possibility that the menstrual phase may have an impact on cortical processing through suppression of negative emotional responses and adult women may have heightened risk of emotional dysregulation in the later stage of their menstrual cycle, although none of the POMS subscales showed significant differences in this study. Perhaps, previous study showed that reduction of beta-endorphin levels and mood variation in the late luteal phase might influence the perception of pain ${ }^{17)}$. Thus, we assume that the fluctuation of gonadal hormone secretion affects the emotional and endogenous pain modulation system during the menstrual cycle and contributes to aggravation of pain under during the luteal phase.

The AA values of both the follicular and luteal phases were significantly decreased between the pre- and post-experimental conditions, though the AA is associated with the sympathoadrenal medullary system and is known to reflect painassociated stress in humans. Previous study has shown that the salivary AA values after stimulating cold pain was significant decreased, compared to those under the pre-experimental condition in Japanese young females ${ }^{7}$. Thus, an increase in AA values in Japanese women before the research might have been caused by the psychological burden of the research itself, and the AA might not be appropriate index to verify acute stress response by heat pain. Also, the psychological data that were obtained using the POMS in the present study were similar during both phases despite evidence of a marked negative emotional state during the luteal phase of the menstrual cycle ${ }^{8,13)}$. Therefore, we need to re-consider the research indexes to the pain 
reaction.

The current study has some limitations. First, the sample size was small, limiting the statistical power of the analysis. Second, classification of the menstrual cycle phases was self-reported, though many relevant studies also did not measure plasma levels of gonadal hormones in order to accurately determine the menstrual cycle phase ${ }^{5,16)}$. Additionally, a previous study reported that measuring the hormone levels was not indispensable for menstrual-cycle evaluation in subjects who had regular menstrual cycles ${ }^{18)}$. Therefore, further studies need to collect samples for more reliable evaluation of gonadal hormones such as estrogen and progesterone. Finally, future studies need to include large-scale trials with both middleaged and advanced-age adults after the menopause, since only young adults participated in this study.

\section{Conclusion}

These findings suggest that Japanese youngadult females are more sensitive to the stimulus of pain during the luteal phase of their menstrual cycle compared to those in the follicular phase. We therefore need to conduct pain management focused on the menstrual cycle and Japanese women of reproductive-age should consider preventative pain management during the luteal phase of their menstrual cycle.

\section{Acknowledgements}

We thank all subjects involved in this study. This study was supported in part by JSPS KAKENHI Grant (22689054 \& 16H05567) from The Ministry of Education, Culture, Sports, Science, and Technology of Japan.
COI: All authors do not have any conflicts of interest to declare for this study.

\section{References}

1) Fillingim, R.B., Maixner, W., Girdler, S.S., Light, K.C., Harris, M.B., Sheps, D.S., Mason, G.A., Ischemic but not thermal pain sensitivity varies across the menstrual cycle, Psychosom. Med., 59 (1997) 512-520.

2) Fillingim, R.B., Ness, T.J., Sex-related hormonal influences on pain and analgesic responses, Neurosci. Biobehav. Rev., 24 (2000) 485-501.

3) Hanci, V., Ayoğlu, H., Yilmaz, M., Yurtlu, S., Okyay, R.D., Erdoğan, G., Başaran, M., Turan, I.O., Effect of menstrual cycle on the injection pain due to propofol, Eur. J. Anaesthesiol., 27 (2010) 425-427.

4) Honca, M., Purtuloglu, T., Honca, T., Sizlan, A., Deniz, S., Kose, A., Ogur, R., Horasanll, E., Effects of the menstrual cycle on injection pain due to rocuronium, J. Clin. Anesth., 25 (2013) 399-402.

5) Iacovides, S., Avidon, I., Baker, F.C., Does pain vary across the menstrual cycle? A review, Eur. J. Pain, 19 (2015) 1389-1405.

6) Jabbour, H.N., Sales, K.J., Smith, O.P., Battersby, S., Boddy, S.C., Prostaglandin receptors are mediators of vascular function in endometrial pathologies, Mol. Cell. Endocrinol., 252 (2006) 191-200.

7) Kakeda, T., Tshikawa, T., Gender differences in pain modulation on nociception by sweet stimulus in adults: a randomized study, Nurs. Health Sci., 13 (2011) 34-40.

8) Lusk, B.R., Carr, A.R., Ranson, V.A., Felmingham, K.L., Women in the midluteal phase of the menstrual cycle have difficulty suppressing the processing of negative emotional stimuli: An eventrelated potential study, Cogn. Affect Behav. Neurosci., 17 (2017) 886-903.

9) Piroli, A., Mattei, A., Carta, G., D’Alfonso, A., Palermo, P., Marinangeli, F., Alfonsi, B., Testa, A., Pasquale, C., Varrassi, G., Paladini, A., Influence of the menstrual cycle phase on pain perception and analgesic requirements in young women undergoing gynecological laparoscopy, Pain Pract., 19 (2019) 140-148.

10) Pogatzki-Zahn, E.M., Mengersen, C., Englbrecht, J.S., Klein, T., Magerl, W., Zahn, P.K., Progesterone relates to enhanced incisional acute pain and pinprick hyperalgesia in the luteal phase of female volunteers, Pain, 21 (2019) (in press).

doi: 10.1097/ j.pain.0000000000001561. [Epub ahead of print] 
11) Riley, J.L., Robinson, M.E., Wise, E.A., Price, D.D., A meta-analytic review of pain perception across the menstrual cycle, Pain, 81 (1999) 225235.

12) Ring, C., Veldhuijzen van Zanten, J.J., Kavussanu, M., Effects of sex, phase of the menstrual cycle and gonadal hormones on pain in healthy humans, Biol. Psychol., 81 (2009) 189-191.

13) Sakaki, M., Mather, M., How reward and emotional stimuli induce different reactions across the menstrual cycle, Soc. Personal Psychol. Compass., 6 (2012) 1-17.

14) Shirasaki, S., Fujii, H., Takahashi, M., Sato, T., Ebina, M., Noto, Y., Hirota, K., Correlation between salivary alpha-amylase activity and pain scale in patients with chronic pain, Reg. Anesth. Pain Med., 32 (2007) 120-123.

15) Stening, K., Eriksson, O., Wahren, L., Berg, G., Hammar, M., Blomqvist, A., Pain sensations to the cold pressor test in normally menstruating women: comparison with men and relation to menstrual phase and serum sex steroid levels, Am. J. Physiol. Regul. Integr. Comp. Physiol., 293 (2007) R1711-R1716.
16) Sherman, J.J., Le Resche, L., Does experimental pain response vary across the menstrual cycle? A methodological review, Am. J. Physiol. Regul. Integr. Comp. Physiol., 291 (2006) R245-R256.

17) Viana, E.S., da Silva, S.B., de Sousa, M.B., Perception of ischemic and pressing pain in young women during menstrual cycle: association with humor and cortisol levels, Acta Cir. Bras., 20 (2005) 220-226.

18) Cimino, R., Farella, M., Michelotti, A., Pugliese, R., Martina, R., Does the ovarian cycle influence the pressure-pain threshold of the masticatory muscles in symptom-free women?, J. Orofac. Pain, 14 (2000) 105-111.

Address for correspondence: Takahiro Kakeda Department of Nursing, Faculty of Nursing, Kansai University of Social Welfare 380-3 Shinden, Ako-city, Hyogo 678-0255, Japan 